\title{
Evaluation of Genetic Counseling Among Cystic Fibrosis Carriers, Michigan Newborn Screening
}

\author{
Mary J. Kleyn, ms, ${ }^{1 *}$ Carrie Langbo, ${ }^{2},{ }^{1}$ Ibrahim Abdulhamid, $\mathrm{MD}^{2}{ }^{2}$ Craig R. Adamski, $\mathrm{Ms},{ }^{3}$ \\ Catherine Allan, ms, ${ }^{4}$ Erin P. Carmany, ${ }^{2},{ }^{5}$ M. Myrtha Gregoire-Bottex, ${ }^{2},{ }^{3}$ \\ Douglas Homnick, MD, MPH, ${ }^{6}$ John Schuen, MD, ${ }^{4}$ and Samya Z. Nasr, $\mathrm{MD}^{7}$
}

\begin{abstract}
Summary. Objective: A quality improvement (QI) strategy to improve the rate of genetic counseling (GC) services was initiated in cystic fibrosis (CF) care Center E in 2010. This statewide study was conducted to determine: (1) GC rates before and after implementation of the QI strategy at Center E; (2) characteristics associated with not receiving GC; and (3) topic areas addressed during GC. Methods: The retrospective study included 1,097 CF carriers born from 2008 to 2011 identified through Michigan's Newborn Screening Program. Rate of GC services was determined for Center $E$ and the other four CF centers before and after the QI change. Bivariate and multivariable logistic regression was used to determine associations between select characteristics and not receiving GC. Topic areas discussed during GC sessions were assessed using frequency tables. Results: Rate of GC services in Center E increased from $23 \%$ in $2008-2010$ to $91 \%$ in 2011 , while at the other centers approximately $92 \%$ received GC services across those years. In 2008-2010, being seen at Center E and black race were significantly associated with increased likelihood of not receiving GC services in adjusted analyses. In 2011, neither characteristic was associated with receipt of GC. Of 16 target topic areas, all were discussed in $85 \%$ of GC sessions. Conclusions: Implementing a QI strategy of providing sweat test results at the GC appointment within Center E resulted in more CF carriers receiving comprehensive GC services. Center-specific procedure differences should be assessed to increase rate of GC services following a positive CF newborn screen. Pediatr Pulmonol. 2013; 48:123-129. ๑ 2012 Wiley Periodicals, Inc.
\end{abstract}

Key words: trait; quality improvement; process; positive screen.

Funding source: none reported.

${ }^{1}$ Michigan Department of Community Health, Lansing, Michigan.

${ }^{2}$ Detroit Medical Center, Children's Hospital of Michigan, Cystic Fibrosis Center, Detroit, Michigan.

${ }^{3}$ Michigan State University Cystic Fibrosis Center, Lansing, Michigan.

${ }^{4}$ Helen DeVos Cystic Fibrosis Care Center, Grand Rapids, Michigan.

${ }^{5}$ Detroit Medical Center, Children's Hospital of Michigan, Division of Genetic and Metabolic Disorders, Detroit, Michigan.

${ }^{6}$ Western Michigan University School of Medicine, Kalamazoo, Michigan.

${ }^{7}$ University of Michigan Cystic Fibrosis Center, Ann Arbor, Michigan.

Conflict of interest: None.

*Correspondence to: Mary J. Kleyn, MS, 201 Townsend Street, Lansing, MI 48909. E-mail: kleynm@michigan.gov

Received 19 June 2012; Accepted 14 August 2012.

DOI $10.1002 /$ ppul.22703

Published online 20 November 2012 in Wiley Online Library (wileyonlinelibrary.com).

(C) 2012 Wiley Periodicals, Inc. 


\section{INTRODUCTION}

Michigan's Newborn Screening (MI-NBS) Program provides an infrastructure for identification of $>50$ serious disorders in infants and management through adulthood. Cystic fibrosis (CF) was added to MI-NBS in 2007. In conjunction with its implementation, MI-NBS established a CF NBS Advisory Board for quality assurance and to pursue research endeavors. It was also established to ensure quality and consistent patient care following a positive NBS. The board is comprised of CF Foundation accredited (CFF) Center Directors, genetic counselors, Department of Community Health Laboratory and NBS Follow-up Program staff, and parents.

CF NBS is currently available in all 50 states and the District of Columbia ${ }^{1}$ through a variety of methodologies. MI-NBS employs a two-tiered immunoreactive trypsinogen (IRT)/DNA algorithm. A positive screen occurs when one or two CF transmembrane conductance regulator (CFTR) mutations are detected following an initial elevated IRT value. The majority of newborns identified are clinically healthy CF carriers. In accordance with the CDC recommendation, ${ }^{2}$ MI-NBS has established an algorithm to provide genetic counseling (GC) services to all parents of newborns scheduled for sweat testing following a positive $\mathrm{CF}$ NBS. For parental convenience, the counseling is usually done the same day the sweat testing is performed.

GC provides information and support to individuals coping with the risk or reality of genetic disorders in their children. Previous studies have shown multiple benefits of GC to families of children following $\mathrm{CF}$ NBS. Farrell et al. ${ }^{3}$ concluded that counseling provided by genetic counselors, pediatric pulmonologists, or nurse specialists was superior to counseling provided by primary care physicians. Parents also prefer receiving information regarding CF NBS from knowledgeable and sensitive specialists. ${ }^{4,5}$ Not only is GC for CF NBS preferred and appreciated by patients, it has also been shown to increase parental knowledge and understanding and reduce anxiety. ${ }^{5-7}$ The goal of GC in MI NBS is to: (1) delineate carrier status versus disease status, (2) discuss the differences between CFTR-related metabolic syndrome and CF, (3) decrease parental anxiety, and (4) discuss and provide carrier testing and preconception planning to parents.

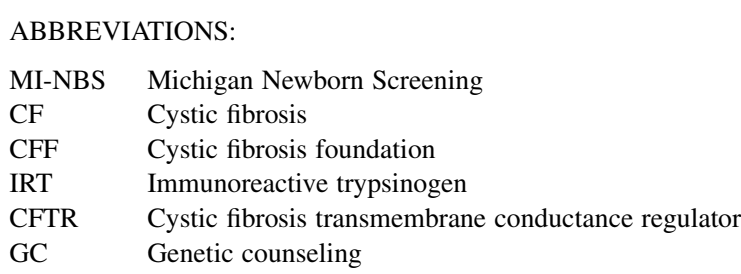

In 2010, the CF NBS Advisory Board recognized a significant disparity in rate of GC services provided by one particular CFF center (Center E) when compared to the state's other four CFF centers (Centers A-D). It was unknown whether certain demographic factors unique to that CFF center's population, logistical barriers, or a combination were contributing to the observed disproportionate number of parents not receiving $\mathrm{GC}$ at that center. This study was conducted to retrospectively evaluate the rate of GC services received by $\mathrm{CF}$ carriers identified through NBS and characteristics associated with not receiving counseling before and after implementation of a QI strategy within Center E. In addition, we assessed topic areas addressed during GC sessions to ensure consistency of quality information provision. This study attempts to identify and reduce any existing barriers to GC for CF NBS and increase efficiency of $\mathrm{GC}$ in the future.

\section{MATERIALS AND METHODS}

MI-NBS is conducted using blood spots collected at birth from infants. Samples with IRT concentrations $>96$ th daily percentile undergo a 40-panel CFTR DNA mutation analysis. Infants with at least one mutation are considered positive for CF NBS and are referred to a CFF Accredited Center for confirmatory sweat testing. Between October 2007 and February 2009, infants with no mutations but IRT concentrations $>99.8$ th percentile were also sent for confirmatory testing.

Families are provided an opportunity for GC on the day of confirmatory sweat testing. In four of Michigan's CFF centers, GC is provided by certified, or active candidate status, genetic counselors. In the remaining center, GC is provided by a physician, either the CF center Director or Associate Director. When parents present for sweat testing in Centers A-D, these centers either require GC in order to receive sweat test results or escort the parents to GC after receiving the results of sweat testing. Center E released the sweat test results to parents before attending the GC clinic visit, which is in a different building. In 2010, Center E implemented a change in policy requiring families receive sweat test results from a genetic counselor at the GC clinic appointment.

Center E took several steps to prepare families for the policy change. When families call to schedule the sweat testing appointment, Center E staff informs them that they will meet with several different providers during the visit and should allow for a lengthy visit. When parents arrive at the sweat test appointment, they are given a written welcome packet which lists all the steps taken during the visit, explains the sweat testing procedure, describes the need for GC on the same day as the sweat test, tells them the genetic counselor will provide 
the sweat test results, and states the time of the GC appointment. This packet also contains walking directions to the GC appointment.

Following GC at all centers, the genetic counselor or CFF center Director completes and submits a standard form with parent demographics, sweat testing results, and key topic areas discussed (from a checklist of 16 benchmarks) to the MI-NBS Program (form available upon request). This form also contains an area to record whether parents refused or failed to attend the GC session.

The study population included infants born between January 1, 2008 and December 31, 2011, identified as positive for CF NBS, and confirmed as CF carriers. Infants whose GC status could not be determined, who received confirmatory testing outside of Michigan, or who received confirmatory testing in-state but not at one of the CFF centers were excluded from analyses.

Data analysis was performed using SAS version 9.2 (SAS Institute, Inc., Cary, NC). Characteristics examined included: birth weight, gestational age, race, neonatal intensive care unit (NICU) admission, maternal age, maternal education, birth year, and CFF center. Since 2007, the linkage of MI-NBS and live births records has been conducted to identify potentially unscreened infants and has a match rate of $>99 \% .^{8}$ Since demographic and perinatal characteristics on birth certificates tend to be more accurately and consistently recorded, we used characteristics from the linked birth certificates instead of from the NBS cards. If a record could not be matched to the birth certificate, characteristics were taken from the NBS card. Gestational age and birth weight were dichotomized into pre-term birth $(<37$ weeks gestation) and low birth weight $(<2,500 \mathrm{~g})$. Race was categorized as white, black, and other. Maternal age at time of birth was calculated from the mother's and infant's birth dates and divided into $<25,25-29,30-34$, and $\geq 35$ years old. Maternal education at time of birth was divided into $<$ high school, completed high school or equivalent, and $>$ high school. Birth years 2008-2010 were collapsed into one group to compare the GC rates before and after implementation of the policy change at Center E. The CFF center responsible for confirmatory testing and GC was retrieved from the NBS follow-up database. Centers A-D were combined into one group to compare the GC rate for those centers with Center E's rate.

The rate of GC uptake was determined for Centers A-D and Center $\mathrm{E}$ for the years before and after the procedural change at Center E. Bivariate and multivariable analyses assessing predictors of not receiving GC were conducted pre- and post-change at Center E. After restricting to those who received or refused counseling as noted on the standard form submitted to the NBS Follow-up Program, bivariate, and multivariable analyses were repeated to assess predictors of refusing counseling. The topic areas discussed during each GC session were assessed using frequency tables. An alpha of 0.05 was used for this study.

\section{RESULTS}

Overall, 460,788 infants born from 2008 to 2011 in Michigan were screened for CF and 1,124 were identified as CF carriers. Twenty-seven CF carriers were excluded from analyses because they were seen at CFF centers outside Michigan $(\mathrm{n}=17)$, at non-CFF centers in Michigan $(n=7)$, or their GC status could not be determined $(n=3)$. Characteristics did not significantly differ between children seen at CFF centers in Michigan and those excluded from the study.

NBS records were linked to birth certificates for 1,095 of the 1,097 children included in the study (99.8\%). Demographic and perinatal characteristics for the study population are presented in Table I. One-third of the study population was seen at Center E.

TABLE I-Demographic and Perinatal Characteristics of Infants Identified as Cystic Fibrosis Carriers, Michigan, 2008-2011

\begin{tabular}{|c|c|c|}
\hline \multirow[b]{2}{*}{ Characteristic } & \multicolumn{2}{|c|}{ Study population } \\
\hline & $\mathrm{N}$ & $\%$ \\
\hline \multicolumn{3}{|l|}{ Birth weight (g) } \\
\hline$<2,500$ & 86 & 7.8 \\
\hline $2,500+$ & 1,011 & 92.2 \\
\hline \multicolumn{3}{|c|}{ Gestational age (weeks) } \\
\hline$<37$ & 111 & 10.1 \\
\hline $37+$ & 986 & 89.9 \\
\hline \multicolumn{3}{|l|}{ NICU } \\
\hline Yes & 73 & 6.8 \\
\hline No & 1,001 & 93.2 \\
\hline \multicolumn{3}{|l|}{ Race } \\
\hline White & 829 & 81.0 \\
\hline Black & 166 & 16.2 \\
\hline Other & 29 & 2.8 \\
\hline \multicolumn{3}{|c|}{ Maternal age (years) } \\
\hline$<25$ & 405 & 36.9 \\
\hline $25-29$ & 323 & 29.4 \\
\hline $30-34$ & 243 & 22.2 \\
\hline $35+$ & 126 & 11.5 \\
\hline \multicolumn{3}{|c|}{ Maternal education } \\
\hline$<$ High school & 154 & 14.1 \\
\hline High school & 317 & 29.1 \\
\hline >High school & 619 & 56.8 \\
\hline \multicolumn{3}{|l|}{ CFF center } \\
\hline Centers A-D & 725 & 66.1 \\
\hline Center E & 372 & 33.9 \\
\hline \multicolumn{3}{|l|}{ Birth year } \\
\hline 2008-2010 & 863 & 78.7 \\
\hline 2011 & 234 & 21.3 \\
\hline Total & 1,097 & 100.0 \\
\hline
\end{tabular}

Excludes those seen outside Michigan (17), seen at non-CFF centers in Michigan (7), and with unknown genetic counseling status (3). 
TABLE II-Genetic Counseling (GC) Rates of Infants Identified as CF Carriers, by Time Period and CFF Center, Michigan, 2008-2011

\begin{tabular}{|c|c|c|c|c|c|c|c|c|}
\hline \multirow[b]{3}{*}{ CFF center } & \multicolumn{4}{|c|}{$2008-2010$} & \multicolumn{4}{|c|}{2011} \\
\hline & \multicolumn{2}{|c|}{ Received GC } & \multicolumn{2}{|c|}{ Did not receive GC } & \multicolumn{2}{|c|}{ Received GC } & \multicolumn{2}{|c|}{ Did not receive GC } \\
\hline & $\mathrm{N}$ & $\%$ & $\mathrm{~N}$ & $\%$ & $\mathrm{~N}$ & $\%$ & $\mathrm{~N}$ & $\%$ \\
\hline Centers A-D & 522 & 89.5 & 61 & 10.5 & 134 & 94.4 & 8 & 5.6 \\
\hline Center E & 63 & 22.5 & 217 & 77.5 & 84 & 91.3 & 8 & 8.7 \\
\hline Summary & 585 & 67.8 & 278 & 32.2 & 218 & 93.2 & 16 & 6.8 \\
\hline
\end{tabular}

Did not receive GC includes no shows (24), active refusals (221), and other (49).

The rate of GC services received was determined by CFF center and birth year category for the 1,097 children who were seen at CFF centers in Michigan with known GC status (Table II). For birth years 2008-2010, approximately $90 \%$ of CF carriers seen at Centers A-D (range, $85-93 \%$ ) received GC compared to $23 \%$ of those seen at Center E. Overall, $68 \%$ of $\mathrm{CF}$ carriers born during these years received GC. In 2011, 94\% of CF carriers seen at Centers A-D (range, 88-100\%) and $91 \%$ of $\mathrm{CF}$ carriers seen at Center $\mathrm{E}$ received GC. The group who did not receive GC included active refusals $(n=221)$, passive refusals through failure to attend GC appointment $(\mathrm{n}=24)$, and missed due to some other reason $(n=49)$.

Table III presents bivariate results for birth years separated into before implementation of the procedural change at Center E (2008-2010) and after (2011). For years 2008-2010, black race, maternal education $<$ high school, and being seen at Center E were significantly associated with increased odds of not receiving GC in bivariate analyses, with being seen at Center E having the strongest association (OR $=29.5,95 \% \mathrm{CI}: 20.0$, 43.4). After adjusting for race, maternal education, and CFF center, only CFF center $(\mathrm{OR}=24.3,95 \% \mathrm{CI}: 16.0$,

TABLE III-Unadjusted Associations Between Select Characteristics and no Genetic Counseling Receipt Among CF Carriers, by Time Period, Michigan

\begin{tabular}{|c|c|c|c|c|}
\hline \multirow[b]{2}{*}{ Characteristic } & \multicolumn{2}{|c|}{ 2008-2010 } & \multicolumn{2}{|c|}{2011} \\
\hline & OR & $95 \% \mathrm{CI}$ & OR & $95 \% \mathrm{CI}$ \\
\hline \multicolumn{5}{|l|}{ Birth weight (g) } \\
\hline$<2,500$ & 1.4 & $(0.8,2.2)$ & 5.7 & $(1.6,20.4)$ \\
\hline $2,500+$ & 1.0 & & 1.0 & \\
\hline \multicolumn{5}{|c|}{ Gestational age (weeks) } \\
\hline$<37$ & 1.5 & $(0.9,2.3)$ & 6.6 & $(2.0,21.7)$ \\
\hline $37+$ & 1.0 & & 1.0 & \\
\hline \multicolumn{5}{|l|}{ NICU } \\
\hline Yes & 1.5 & $(0.9,2.6)$ & 4.6 & $(1.1,18.9)$ \\
\hline No & 1.0 & & 1.0 & \\
\hline \multicolumn{5}{|l|}{ Race } \\
\hline White & 1.0 & & 1.0 & \\
\hline Black & 5.8 & $(3.9,8.5)$ & 2.3 & $(0.7,7.9)$ \\
\hline Other & 1.1 & $(0.4,3.2)$ & 1 & \\
\hline \multicolumn{5}{|c|}{ Maternal age (years) } \\
\hline$<25$ & 1.0 & $(0.7,1.4)$ & 0.7 & $(0.2,2.5)$ \\
\hline $25-29$ & 1.0 & $(0.7,1.5)$ & 0.3 & $(0.1,1.8)$ \\
\hline $30-34$ & 1.0 & & 1.0 & \\
\hline $35+$ & 0.9 & $(0.6,1.6)$ & 1.3 & $(0.3,6.0)$ \\
\hline \multicolumn{5}{|l|}{ Maternal education } \\
\hline$<$ High school & 1.7 & $(1.1,2.6)$ & 1.4 & $(0.4,5.3)$ \\
\hline High school & 1.1 & $(0.8,1.6)$ & 0.7 & $(0.2,2.7)$ \\
\hline$>$ High school & 1.0 & & 1.0 & \\
\hline \multicolumn{5}{|l|}{ CFF center } \\
\hline Centers A-D & 1.0 & & 1.0 & \\
\hline Center E & 29.5 & $(20.0,43.4)$ & 1.6 & $(0.6,4.4)$ \\
\hline
\end{tabular}

${ }^{1}$ Unstable model. 
$37.1)$ and race $(\mathrm{OR}=1.7,95 \% \mathrm{CI}: 1.0,2.9)$ were significantly associated with lack of GC (data not shown). For birth year 2011, low birth weight, pre-term birth, and being admitted to the NICU were significantly associated with increased likelihood of not receiving GC. Being seen at Center $\mathrm{E}$ and black race were not significantly associated with GC services received in 2011.

After excluding CF carriers who did not show up for the scheduled GC appointment or did not receive counseling for some other reason, the bivariate associations between characteristics and refusing GC were determined. The associations were similar to those assessing failure to receive $\mathrm{GC}$ for any reason. For birth years 2008-2010, black race, maternal education $<$ high school, and being seen at Center E were all significantly associated with increased likelihood of refusing GC in unadjusted models (data not shown). After adjustment, only being seen at Center $\mathrm{E}$ remained significantly associated with refusal. For birth year 2011, pre-term birth and NICU admission were significantly associated with GC refusal in bivariate models.

Of the $803 \mathrm{CF}$ carriers who received GC, information was recorded on the GC form for 795 children (99\%). Mothers were present for $90 \%$ of the sessions and fathers for $70 \%$. Two family members attended the counseling session for nearly three-quarters of the carriers $(71 \%)$. The 16 content area items included in the GC form were covered in nearly all of the sessions (Table IV). Discussion of gene sequencing took place in $91 \%$ of sessions, with all other topic areas discussed in more than $97 \%$ of sessions. All 16 items were discussed in $85 \%$ of sessions.

TABLE IV-Summary of Genetic Counseling Information Discussed With Families of CF Carriers, Michigan, 2008-2011

\begin{tabular}{ccc}
\hline & $\mathrm{N}$ & $\%$ \\
\hline Section I & & \\
A & 786 & 98.9 \\
B & 786 & 98.9 \\
C & 780 & 98.1 \\
D & 784 & 98.6 \\
E & 773 & 97.2 \\
Section II & & \\
A & 786 & 98.9 \\
B & 785 & 98.7 \\
C & 784 & 98.6 \\
D & 781 & 98.2 \\
E & 775 & 97.5 \\
Section III & & \\
A & 785 & 98.7 \\
B & 786 & 98.9 \\
C & 776 & 97.6 \\
D & 775 & 97.5 \\
E & 725 & 91.2 \\
F & 769 & 96.7 \\
\hline
\end{tabular}

Form available upon request.

\section{DISCUSSION}

GC has been recommended as part of NBS programs for many diseases including $\mathrm{CF}^{2,3}$ The current literature on CF NBS establishes the benefits of GC from both the parents' and providers' perspective. Almost all of the reported studies have relied upon parental report focusing on methods of communication, recall of information by parents and types of information given to parents. ${ }^{4-7}$ GC was found to be very effective in improving parental knowledge about carrier status of the parents and their children, presence or absence of disease in the screened child, risk of having future children with $\mathrm{CF}$, and future family planning decisions. ${ }^{9}$ However, although there is a reduction in parental anxiety about abnormal CF NBS test with GC, many parents were still confused about the newborn's carrier status and concept of residual risk even after GC. ${ }^{5}$

While assessing parental viewpoints of $\mathrm{GC}$ in $\mathrm{CF}$ NBS is certainly helpful, further assessment of GC efficacy and topic areas addressed is needed using reports from physicians and genetic counselors. MINBS requires submission of GC reporting forms developed by MI genetic counselors to ensure those providing GC uphold practice based competencies established by the American Board of Genetic Counseling. ${ }^{10}$ This standardized approach ensures all parents are afforded consistent comprehensive information, and collected information is reviewed by the CF NBS Advisory board to encourage compliance with benchmarks established for optimal service. Sixteen benchmarks are included on the GC form and ensure the genetic counselor obtains a three generation pedigree with targeted CF questions; assesses psychosocial needs; discusses basic genetic concepts (autosomal recessive inheritance); explains implications of carrier results for the child, parents and extended family members; offers parental carrier screening and provides written educational information. In all five CFF centers, nearly all 16 benchmarks were achieved, providing high degree of consistency of GC services between CFF centers in the State of Michigan CF NBS program. Thus, the most critical component to receipt of comprehensive GC services in Michigan is ensuring that parents of $\mathrm{CF}$ carriers attend the GC appointment.

Overall, there has been limited literature evaluating variables influencing the rate of GC services that assess impact of both potential logistical barriers and demographic factors. Our study assessed the rate of GC in different CFF centers across the state to identify and potentially eliminate barriers to GC. Centers A-D either have always required $\mathrm{GC}$ in order to receive sweat test results or escorted parents to $\mathrm{GC}$ after receiving the results of sweat testing which has led to the greatest 
receipt of GC services, while Center E had significantly reduced compliance with GC during 2008-2010 probably due to lack of parental understanding of the importance of GC or logistical barriers. Following a change of policy in Center $\mathrm{E}$ to require parents obtain GC in order to receive sweat test results, there were similar acceptance rates of GC services among all five CFF centers in 2011, with $93 \%$ of parents of CF carriers receiving GC.

The change in the rate of GC services seen in Center $\mathrm{E}$ is in agreement with Comeau et al. ${ }^{11}$ who reported the results of three models of post-screening communication with families at five CF centers in Massachusetts. The authors found 95\% compliance with sweat testing but a wide variability with completion of GC (32$90 \%$ ). Families who were escorted to GC at the same day of sweat chloride testing were more likely to have GC (84-90\%) than those who scheduled GC independently of the sweat test and usually at a different date (32\%).

Although not addressing the rate of GC directly, Lagoe et al. ${ }^{12}$ evaluated the rate of parental CF carrier screening by randomizing families into two groups; one having GC on the same day as the sweat test (referred to as Special Care) versus reporting sweat test results via phone by a pediatrician who may have recommended GC at a later date (referred to as standard care). Fifty percent (17/34) of the parents who were managed via special care had parental $\mathrm{CF}$ carrier screening versus $22 \%(6 / 27)$ of the parents who were managed via standard care approach. The authors concluded that offering GC and carrier genetic testing increases probability of parental consent to further identification of their carrier status and may facilitate further dissemination of such knowledge and testing among relatives.

Our study highlights the importance of comparing center-specific differences when assessing the rate of GC services. Without this, black race would have been most significantly associated with not receiving GC in unadjusted and adjusted analyses during years 20082010. Since $36 \%$ of Center E's patient population was black compared to $4-11 \%$ of Center A-D's populations, the significant finding for black race was most likely a spurious association. Center E's initial policies for GC services had more influence on rate of GC than race. Thus, the center-specific procedures affected GC rates more than demographic characteristics. To ensure the highest rate of GC, each CFF center should evaluate the rate of GC for CF NBS cases and its policies for providing sweat test results.

Tracking when procedural changes are implemented is critical for assessing the effect of those changes and associations before and after implementation. Our study found that the $\mathrm{GC}$ rates among $\mathrm{CF}$ carriers statewide increased from $68 \%$ for $2008-2010$ to $93 \%$ in 2011 , largely driven by Center E's rate improving from $23 \%$ to $91 \%$. After repeating the bivariate analyses for all years combined (2008-2011), the following variables were significantly associated with lack of GC receipt: low birth weight, pre-term birth, NICU admission, black race, and maternal education $<$ high school. The separate analyses for the years before and after implementation of the quality improvement project at Center $E$ indicated that black race and maternal education were associated with reduced GC receipt before implementation. The perinatal health complications such as low birth weight, pre-term birth, and NICU admission were associated with reduced GC receipt after implementation. Potential precarious health status of these infants may impact parental uptake of GC, but these results should be interpreted with caution since only 16 CF carriers born in 2011 did not receive GC.

Our study provides additional information regarding both the type of information provided in a CF NBS GC session as well as factors that impact the rate of GC for CF carriers identified through NBS. We have shown that families in the State of Michigan receive GC services in a uniform manner and highlight the importance of an advisory board in program evaluation and improvement. As seen in other studies, we show further evidence that the best service delivery model is one that offers GC on the same day as the sweat test in a NBS population. Unlike previous studies, however, we demonstrate that just offering GC on the same day as the sweat test may not be enough to ensure that families receive these services. Parents who are worried primarily about their child being affected with CF may not consider GC a priority at the time of the sweat test. If they receive negative sweat test results before receiving counseling, they may not have full understanding of the importance of GC, may not be concerned about future family planning given the recent birth of a baby, or simply may not have been educated about the time needed for the two appointments on the same day. This may cause them to defer having GC on the day of the appointment if given a choice and fail to follow up at a later date after receiving the reassurance of a negative sweat test result. This may ultimately suggest that more pre-sweat test education is needed regarding the benefits of GC in the CF NBS population.

\section{REFERENCES}

1. National Newborn Screening and Genetics Resource Center. National Newborn Screening Status Report. 2012; Available from: http://genes-r-us.uthscsa.edu/nbsdisorders.htm.

2. Grosse SD, Boyle CA, Botkin JR, Comeau AM, Kharrazi M, Rosenfeld M, Wilfond BS, CDC. Newborn screening for cystic fibrosis: evaluation of benefits and risks and recommendations for state newborn screening programs. MMWR Recomm Rep 2004;53:1-36. 
3. Farrell M, Certain L, Farrell P. Genetic counseling and risk communication services of newborn screening programs. Arch Pediatr Adolesc Med 2001;155:120-126.

4. Tluczek A, Koscik RL, Modaff P, Pfeil D, Rock MJ, Farrell PM, Lifchez C, Freeman ME, Gershan W, Zaleski C, Sullivan B. Newborn screening for cystic fibrosis: parents' preferences regarding counseling at the time of infants' sweat test. J Genet Couns 2006;15:277-291.

5. Lang CW, McColley SA, Lester LA, Ross LF. Parental understanding of newborn screening for cystic fibrosis after a negative sweat-test. Pediatrics 2011;127:276-283.

6. Ciske DJ, Haavisto A, Laxova A, Rock LZ, Farrell PM. Genetic counseling and neonatal screening for cystic fibrosis: an assessment of the communication process. Pediatrics 2001;107:699705.

7. Cavanagh L, Compton CJ, Tluczek A, Brown RL, Farrell PM. Long-term evaluation of genetic counseling following falsepositive newborn screen for cystic fibrosis. J Genet Couns 2010;19:199-210.
8. Korzeniewski SJ, Grigorescu V, Copeland G, Gu G, Thorburn KK, Rogers JD, Young WI. Methodological innovations in data gathering: newborn screening linkage with live births records, Michigan, 1/2007-3/2008. Matern Child Health J 2010;14: 360-364.

9. Dillard JP, Tluczek A. Information flow after a positive newborn screening for cystic fibrosis. J Pediatr 2005;147:S94S97.

10. American Board of Genetic Counseling. Practice-Based Competencies. 2006; Available from: http://www.abgc.net/docs/Practice\%20Based\%20Competencies_Aug\%202006\%2010-29-09.pdf.

11. Comeau AM, Parad R, Gerstle R, O'Sullivan BP, Dorkin HL, Dovey M, Haver K, Martin T, Eaton RB. Communications systems and their models: Massachusetts parent compliance with recommended specialty care after positive cystic fibrosis newborn screening result. J Pediatr 2005;147:S98-S100.

12. Lagoe E, Labella S, Arnold G, Rowley PT. Cystic fibrosis newborn screening: a pilot study to maximize carrier screening. Genet Test 2005;9:255-260. 